\title{
The Design Research of the Embedded Control System of Industrial Robots
}

\author{
Jiuchao Li, Cheng Yao, Haifeng Xu \\ Engineering Technology Institute of Changchun, China \\ 247658766@qqcom, 317456421@qqcom, 3174296251@qqcom
}

Keywords:modular robots; embedded systems; control system

\begin{abstract}
. based on modular robot control system reconstruction problem, put forward a kind of flexible embedded control system architecture. The control system of various hardware and software function module is extracted into a standard components, hardware module adopted the dual-core processor is designed in order to meet the needs of computing power and miniaturization, between modules communicate through standard of field bus interface. The embedded control system has been applied in six joint industrial robot and the experimental results show that it can adapt to changes in the robot mechanical structure and the tasks and make the system greatly shorten the development cycle.
\end{abstract}

\section{Introduction}

As car, rail transportation, electric power equipment manufacturing, electronic information and other important areas of industrial robot application scope expands, industrial robot can complete a variety of different tasks in a complex environment, when work tasks and environment change robot should have a certain ability to adapt. Therefore, people put forward the concept of modular industrial robot, through the modular design features, can be rapidly reconstructed to meet the actual needs of robot system, which can greatly shorten the development cycle of the robot.

Modular industrial robot research caused the attention of the academia, it puts forward new requirements for the design of the control system, must solve system of reconfigurable architecture, design of module library and so on. At present, many scholars studied the modular control structure of the industrial robot. Stewart et al., this paper proposes a dynamic reconfigurable real-time software design based on port object framework; Rossmann etc. This paper proposes a multi-agent based modular control system architecture (Agent); Qiu-xuan wu etc. Put forward a kind of control structure based on multi-agent distributed system; Rules is proposed based on a module free libraries and macro motion heuristic planning methods. In recent years at home and abroad for the development of common industrial robot control system based on PC platform, this paper proposes a flexible architecture is used to solve the problem of modular embedded system reconstruction of industrial robot, this paper gives the system of hard and soft components, reconstruction method, and the realization of the software component technology.

\section{Robot Flexible Architecture of the Embedded Control System}

A. reconfigurable hardware logical structure

A reconfigurable robot control system allows the user to according to the need for matching and integration, to change or extend the functionality of system, in order to rapidly adapt to different application requirements, the architecture should have the following properties: interoperability, portability, scalability, and replaceability.

For freedom of the industrial robot function module static configuration, control system hardware is encapsulated into several basic functional components, including: motion control template, logic control template, teaching modules, etc., through Ethernet and CAN bus connection between hardware components.

Motion control module chooses TI company OMAPLl38 dual-core processor chip, it integrates 
TMS320C674x DSP kernel and ARM926EJ S dual-core, has characteristic of high performance and low power consumption. ARM kernel complete non real-time instruction interpretation, trajectory planning, data management, task management, network communication etc, DSP kernel due to a strong ability to complete interpolation calculation, inverse kinematics, speed and position control and the I/O control, real-time tasks. Position control of the FPGA chip contains such as interpolation, position PID circuit, adopted EPIC3T144 chip of ALTERA company, by loading different programs to achieve functional reconstruction. In order to increase the speed of multi-axis linkage interpolation, interpolation algorithm adopt soft interpolation (coarse) ten hard interpolation (fine) combined with pattern, hard interpolation based on digital multiplier. The module is connected to the drive interface provides two forms: digital pulse interface, industrial Ethernet interfaces, industrial Ethernet interface can be compatible with a variety of communication protocols of the drive, has realized the Mecha - trolink III agreement, it is put forward by the Japan yaskawa company a EEE802.3 u standard Ethernet protocol, transmission speed can reach 100 MBPS. OMAPLl38 dual-core processor chip can provide abundant peripheral interface, including Ethernet, USB and RS232 interface, through the Ethernet interface module and motor control module of communication teaching. Teaching module by OMAPLl38 dual-core processor circuit, 48 buttons and a 5.7 -inch, $640 \times 480$ color TFr of buttons including edit button, the manual operation key, teaching programming key and function keys. In order to ensure the functional reconstruction has nothing to do with the hardware platform, using the Windows CE6.0 operating system. This module contains the software function component are: file management, teaching programming, parameters setup, management, graphical simulation, Ethernet communication, etc. $\mathrm{I} / \mathrm{O}$ module is responsible for the logic control functions, to realize the harmonious operation of robot and peripheral equipment. CAN protocol is based on international standards organization open systems interconnection model, simple protocol, the highest communication rate of $1 \mathrm{Mbit} / \mathrm{s}$, direct transmission distance up to $10 \mathrm{~km}$, take more main works, electromagnetic interference resistance, error correction ability strong; At the same time, the CAN interface convenient installation, low cost. This paper selects the CAN bus as the motion controller and the way of data communication between $\mathrm{I} / \mathrm{O}$ templates.

B. industrial Ethernet communication protocols

Robot teaching module with Ethernet communication function of the motion control module USES the Socket (the Sock - et) way to do this, the Socket interface is divided into two parts: client and server, this teaching module as the client, motion control module as a server. This paper USES the communication mode USES the master-slave mode, the teaching module to active, sending the robot motion program, control parameters and command word, is used to control the movement of the robot movement, and movement control module in real time will coordinate data and state parameter to the teaching module. At the start of communication, will be the first to shake hands. The data link layer protocol defines the command and data message form, command message is used to control the robot's movement and the process, defined as a standard message format; Data packets used to transmit the robot's movement process and parameters, the data quantity is large, defined as an extension of packet format. There are 7 bytes standard message format, the first byte is a message identifier, to identify a communication, this article set for "\%". 2 bytes are message types, is used to identify the transmission of message type and processing mode, the message types defined as: command, data, and provides two kinds of message processing mode: real-time and non real-time. When need robot to complete stop treatment, reduction processing and distance alarm tasks, such as packet is transmitted with real-time identification; The robots to general processing tasks, the message identifier for the real time. The length of the message by byte identifies 3 and 4 , up to 65536 bytes. In bytes for the data segment, 5 and 6 to 7 bytes with "\&" to identify the message. When type identification for the data transmission of information, using an extension of message format, the data length can reach $2 \mathrm{~K}$ bytes. Teaching modules according to the communication protocol message will need to send data encapsulation, and then use the data transmission function next to the motion control module, including program, parameters, command word. 


\section{Industrial Robot Control Software Reconfiguration Method}

Modular robot control software should have cut sex and multiplicity, the whole architecture should be able to be reconfigured, to satisfy the needs of a variety of applications, has the ideal concept of "openness" control software should be broken up into multiple standard parts. In order to realize the convenient and at the same time satisfy the configuration features, this paper proposes a flexible software framework structure. "Soft" and "openness" these two concepts have differences, but also has the same features, focusing on "openness" and external system by defining a standard interface operation, and the "soft" refers to the system can be changed by its own structure to adapt to the external environment. This kind of flexible control system adopts component-based configuration structure, its software consists of three parts: embedded flexible control system development platform, robot function component library and motion planning and control algorithm component library? Flexible embedded control system development platform is used to implement functional component packaging, such as system configuration tasks. For modular robot control system has the characteristics of multifunctional and multiple objects, and the overall structure of the control software flow is designed to be at the front desk, backstage program module, the background module program, also known as the "background" is mainly used to complete the control instruction preparation and parameter management, front desk module is a cycle to execute programs running, it is the core of the whole control system. Used in the process of system operation in real time interrupt service program output, Taiwan before and after modules work together to complete all the control system of robot control and management tasks. Program mode of function components specified by the configuration script file. The extraction of componentbased structure is the key component, namely to refine the reusable objects. Through well defined interfaces between these objects communicate with each other, can be the basic object or will they formed after further analysis of particle size smaller objects, in the development process to reuse them. This paper set up the component library is composed of component library user layer and core layer component library, through the standard hardware interface for communication between. User layer component library including man-machine interface, teaching programming, motion planning, parameter management, and other non real-time component library. Because the user layer using the Windows CE operating system, software module USES the COM component. Core layer component library including decoding process, speed control, position control, kinematics and inverse kinematics of the robot operation of real-time tasks. As core layer did not use the operating system, this paper make use of the functions implemented. Various functional components according to the standard interfaces encapsulate functional component interfaces.

\section{Component Library Design Control Model and Algorithm}

Flexible modular robot control system is largely dependent on the performance of control model and algorithm, developed the model of algorithm components include: instruction decode, robot kinematics and inverse kinematics, speed control, joint interpolation, closed loop position control, etc. Model algorithm in the implementation are encapsulated into a standard form of function:

The name of the function parameters (parameter $1, \ldots, \mathrm{n}$ )

IF (Event - state $==$ False) return;

Parameter_l;

Parameter_n;

Method;

Address_map;

Among them, the Event - the state is used to set up a functional component activation condition, (Parameter_1,..., the Parameter - n) said the connection relations between the components and interface definition, configuration Settings and initialized. Method according to program written in $\mathrm{C}$ language model and the algorithm, including basic statement and function. Address-the role of the map parameter function components from the hardware and programming environment, when used in specific binding of specific physical Address again. 
Mainly by the decoding process, rectangular coordinate calculation, interpolation arithmetic, inverse kinematics, deceleration, position control modules, etc. The meaning of the parameters in the figure is: ( $\mathrm{x}, \mathrm{y}, \mathrm{z}, \mathrm{U}, \mathrm{y}$, shape) said the coordinate data after decoding, $\mathrm{Q}$ as a sign coordinate system selection, and as the command speed, Type, as the movement way sign, (/ $3 \mathrm{~d}, \mathrm{y})$ and (P, $\mathrm{P}$, and $P$, respectively) of the robot end position and posture, $(Q ., j, Q:, j ., \ldots, Q ., j)$ as the first, a joint coordinates of interpolation cycle. The function of the rectangular coordinates calculation module for using robot kinematics joint coordinate transformation for the position and posture of robot end. The function of the interpolation module is based on trajectory start-stop movement way, coordinates and parameters such as speed, joint coordinate system or right-angle coordinates of interpolation arithmetic. Interpolation is calculated under linear coordinate of the robot end position and attitude, need to use the inverse kinematics transform it into the corresponding joint coordinates, to control the movement of the servo system.

\section{A. robot kinematics artifacts}

Robot kinematics components are used to implement the robot joint coordinate system to the coordinates of the end of the robot coordinate transformation between the position and posture. The component is encapsulated as struct Robot - T6 CAL - T6 (struct Robot_joints * q), inlet parameters of Robot joint coordinates (ql, q2,..., q6) (struct Robotjoints * q), export parameters of the position and posture of Robot end T6 (struct Robot - T6:\} : T6), public interface parameters for Robot Denavit Hartenberg - (d.h.) parameters, namely the joint Angle, torsional Angle, connecting rod length and offset, etc.

Method in order to establish the principle of d.h. according to robot coordinate system, establish A link coordinate system, using DH connecting rod parameter calculation of adjacent A transformation matrix between the connecting rod. $(I=1,2, \ldots, 6)$, a term used to describe the adjacent coordinate transformation relation between the connecting rod coordinate system. Through the connecting rod transformation matrix multiplication, robots will be given at the end of the transformation matrix.

B. the inverse kinematics of artifacts

The end of the robot inverse kinematics components used to implement the robot position and attitude to the coordinate mapping between the coordinates of the robot joint, which was carried out on the end of the robot path planning, to achieve accurate control of robot overall motion. The component is encapsulated as struct Robot joints Robot - rev (struct Robot - T6 * T6, struct Robot_joints * q BK), population parameters for the position and posture of Robot end T6 (STMCT Robot_T6* T6), export parameters for the current position of the Robot joint coordinates (q1, q2,..., q6) (struct Robot - joints * q), d-h parameters is public interface parameters for the Robot, Robot minimum allowed each joint coordinates, and the maximum allowable coordinates.

Based on transformation matrix of robot end method, analytic method is used to solve the joint coordinates. Due to the inverse kinematics to solve multiple solution problems, according to the scope of robot movement to determine, eliminate inaccessible. The current track points calculated six joint coordinates) and the position of a joint coordinates qi\&l comparison, choose the smallest \%o value as the desires of the joint coordinates.

\section{C. decoding processing components}

Decoding component's function is to transmit the various robot teach box next to decode, decoding results in the first place in the first level cache, and according to the command to do the corresponding pretreatment, such AS long calculation, radius of circular arc processing, and then after BS register finally decoding to register AS implementation, constitute a three-level cache structure, preparing for the final instruction execution.

\section{Robot Control System Field Experiment and Result Analysis}

Flexible use of the proposed embedded control system development platform, application experiment was carried out in some model robot joints. Field experiment environment, mobile robot control system is shown in figure 1. 


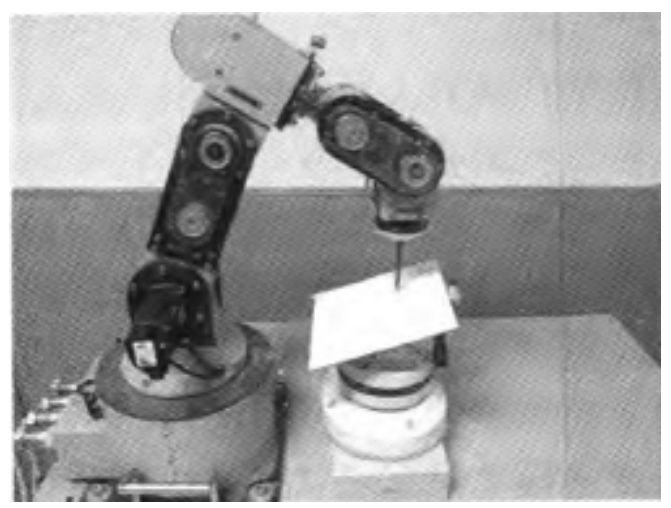

Fig.1 Real experiment environment of robot control system

Robot control system hardware of six axis motion control module, teaching module and I/o modules, including man-machine interface, control software programming, parameter setting, teaching interpolation calculation, decoding process, speed control, position control, kinematics and inverse kinematics, and other functions, for the robot control system software to write configuration files, topology between components are presented. Developed by the robot control system of the teaching module interface, as shown in figure 2.

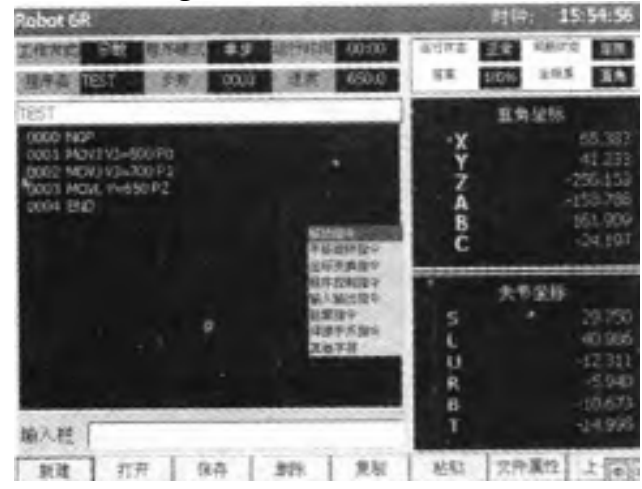

Fig.2 Teaching modular interface of six-joint robot

Without robot ontology trajectory position when the maximum error is $0.08 \mathrm{~mm}$, maximum error is 0.2 , with ontology trajectory position when maximum error is $0.1 \mathrm{~mm}$, maximum error of 0.6 . The model trajectory position the theoretical value, permissible error is $0.1 \mathrm{~mm}$, so it can meet the requirements of the model in the robot control precision. Robot control system after a long run, the results show that the embedded flexible control system can increase the openness and scalability of the robot system, the hardware and software configuration is convenient, high reliability control system.

\section{Conclusion}

In view of the actual demand for modular robot control system, this paper proposes a control system of flexible structure, it can solve the reconfigurable robot control function of embedded type system problem, the previous study of modular robot control system is mainly based on the PC platform. The robot control system hardware and software function module is extracted as a standard component, in order to satisfy the robot to computing power and miniaturization of the control system request, the hardware module adopted dual-core processor and the use of communication between the fieldbus standard interface implementation. Robot control software adopts component-based configuration structure, it consists of the flexible embedded control system development platform, robot function component library and motion planning and control algorithm of component library of three parts, all software modules according to the standard interface for encapsulation. Developed control system in six joint module is carried out on the industrial robot application, field experiments show that the system performance is stable and reliable, able to adapt to the change of the modular robot mechanical structure and the tasks demand, can greatly shorten the development cycle. 


\section{References}

[1] Cao Y,E1Maraghy H,Azab A. Reconfigurable Control Structure for Robots in Assembly[J].Journal of Intelligent and Robotic Systems,2007,(04):419-439.

[2] Stewart D B,Volpe R A,Khosla P. Design of dynamically reconfigurable real-time software using port-based objects[J].IEEE Transactions on Software Engineering,1997,(12):759776.doi:10.1109/32.637390.

[3] Faí(n)a A,Bellas F,Souto D. Towards an evolutionary design of modular robots for industry[A].La Palma,Canary Islands,Spain,2011.

[4] Fitch R,Rus D. Self-reconfiguring robots in the USA[J].Journal of Japanese Society of Robotics,2003,(08):832-838.

[5] Fukuda T,Nakagawa S,Kawauchi Y. Structure decision method for self organizing robots based on cell structures-CEBOT[A].Scottsdale,AZ,USA,1989.695-700.

[6] Schmitz D,Khosla P,Kanade T. The CMU reconfigurable modular manipulator system[M].Pittsburgh,PA,USA:Carnegie Mellon University,1988.

[7] Aspragathos N A. Reconfigurable robots towards the manufacturing of the future[A].2005.

[8] Murata S,Yoshida E,Kamimura A. M-TRAN:self-reconfigurable modular robotic system[J]. IEEE/ASME Transactions on Mechatronics,2002,(04):431-441.

[9] Yim M,Shen W,Salemi B. Modular self-reconfigurable robot systems-challenges and opportunities for the future[J].IEEE Robotics and Automation Magazine,2007,(01):43-52. 